\title{
ASYMPTOTIC EXPANSIONS OF THE SOLUTIONS TO THE HEAT EQUATIONS WITH HYPERFUNCTIONS INITIAL VALUE
}

\author{
Kunio Yoshino and Yasuyuki OKa
}

Abstract. We will derive the asymptotic expansions of solutions of the heat equation with hyperfunctions initial data.

\section{Introduction}

In [4], T. Matsuzawa characterized hyperfunctions with compact support as the initial value of the solutions to the heat equations as follows:

Theorem 1 ([4]). Let $u$ be a hyperfunction with compact support $K$. Then $U(x, t)=(u * E)(x, t)$ satisfies

(i) $\left(\frac{\partial}{\partial t}-\Delta\right) U(x, t)=0$,

(ii) $U(x, t) \in C^{\infty}\left(\mathbb{R}^{d} \times(0, \infty)\right)$,

(iii) For any $a>0$, there exists $C_{a}>0$ such that

$$
|U(x, t)| \leq C_{a} e^{a / t}, x \in \mathbb{R}^{d}, 0<t<1,
$$

(iv) For any $\delta>0, U(x, t) \longrightarrow 0$ uniformly in $\left\{x \in \mathbb{R}^{d} \mid \operatorname{dis}(x, K) \geq \delta\right\}$ as $t \longrightarrow 0$ and

(v) $U(x, t) \rightarrow u, t \rightarrow 0$ in $\mathcal{A}^{\prime}(K)$, i.e.,

$$
\langle u, \varphi\rangle=\lim _{t \rightarrow 0} \int_{\mathbb{R}^{d}} U(x, t) \chi(x) \varphi(x) d x
$$

for any entire functions $\varphi$, where $E(x, t)=(4 \pi t)^{-\frac{d}{2}} e^{-\frac{x^{2}}{4 t}}, x \in \mathbb{R}^{d}$, $0<t<+\infty$ and $\chi \in C_{0}^{\infty}\left(\mathbb{R}^{d}\right)$ such as $\chi=1$ in a neighborhood of $K$. Conversely, if every $C^{\infty}$-function $U(x, t)$ defined in $\mathbb{R}^{d} \times(0, \infty)$ satisfies (i), (iii) and (iv), then there uniquely exists $u \in \mathcal{A}^{\prime}(K)$ such that $U(x, t)=(u * E)(x, t)$.

Received April 22, 2008.

2000 Mathematics Subject Classification. 32A45, 35B05, 35K05, 46F15.

Key words and phrases. heat equation, heat kernel, hyperfunctions, Fourier-hyperfunctions, Gel'fand-Shilov space. 
We analyze $(\sharp)$ more precisely. Formally, we have the following formal calculation:

$$
\begin{aligned}
U(x, t) & =\left(\frac{1}{2 \pi}\right)^{d} \int \hat{U}(\xi, t) e^{i x \cdot \xi} d \xi \\
& =\left(\frac{1}{2 \pi}\right)^{d} \int \hat{u}(\xi) \hat{E}(\xi, t) e^{i x \cdot \xi} d \xi \\
& =\left(\frac{1}{2 \pi}\right)^{d} \int \hat{u}(\xi) e^{-t \xi^{2}} e^{i x \cdot \xi} d \xi \\
& =\left(\frac{1}{2 \pi}\right)^{d} \int \hat{u}(\xi) \sum_{k=0}^{\infty} \frac{\left(-t \xi^{2}\right)^{k}}{k !} e^{i x \cdot \xi} d \xi \\
& =\left(\frac{1}{2 \pi}\right)^{d} \sum_{k=0}^{\infty} \frac{t^{k}}{k !} \int \hat{u}(\xi)\left(-\xi^{2}\right)^{k} e^{i x \cdot \xi} d \xi \\
& =\sum_{k=0}^{\infty} \frac{t^{k}}{k !} \Delta^{k} u,
\end{aligned}
$$

where $\hat{f}$ is the Fourier transform of $f$. For example, we have $E(x, t) \sim$ $\sum_{k=0}^{\infty} \frac{t^{k}}{k !} \Delta^{k} \delta$, where $\delta$ is Dirac's delta function.

As a consequence of our strict consideration on this formal calculation, we will derive the asymptotic expansions of solutions of the heat equation with hyperfunctions with compact support initial data as follows:

Main Theorem 1. Let $U(x, t) \in C^{\infty}\left(\mathbb{R}^{d} \times(0, \infty)\right)$ satisfying the following conditions:

(i) $\left(\frac{\partial}{\partial t}-\Delta\right) U(x, t)=0$

(ii) $\forall \epsilon>0, \exists C_{\epsilon} \geq 0$ s.t. $|U(x, t)| \leq C_{\epsilon} e^{\varepsilon / t}, 0<t<1, x \in \mathbb{R}^{d}$,

(iii) $\forall \delta>0, U(x, t) \rightarrow 0$ uniformly in $\left\{x \in \mathbb{R}^{d} \mid \operatorname{dist}(x, K) \geq \delta\right\}$ as $t \rightarrow 0$.

Then $U(x, t)$ has the following asymptotic expansion:

$$
U(x, t) \sim \sum_{k=0}^{\infty} \frac{t^{k}}{k !} \Delta_{x}^{k} u,\left(u \in \mathcal{A}^{\prime}(K), \text { where } u=\lim _{t \rightarrow 0} U(x, t)\right) .
$$

That is, for any entire function $\varphi$,

$$
\lim _{t \rightarrow 0+}\left|\int_{\mathbb{R}^{d}} U(x, t) \chi(x) \varphi(x) d x-\left\langle\sum_{k=0}^{\frac{N}{2}} \frac{t^{k}}{k !} \Delta_{x}^{k} u, \chi \varphi\right\rangle\right| t^{-\frac{N}{2}}=0,
$$

where $\Delta_{x}=\partial_{x_{1}}^{2}+\cdots+\partial_{x_{d}}^{2}$ and $\chi(x) \in C_{0}^{\infty}\left(\mathbb{R}^{d}\right)$ such as $\chi=1$ in a neighborhood of $K$. 


\section{Asymptotic expansions of the solutions to the heat equations with hyperfunctions with compact support initial value}

We will derive the asymptotic expansions of the solutions of the heat equations with hyperfunctions with compact support initial value in this section. First of all, we fix some notations. We use a multi-index $\alpha \in \mathbb{Z}_{+}^{d}$, namely, $\alpha=\left(\alpha_{1} \cdots \alpha_{d}\right)$, where $\alpha_{i} \in \mathbb{Z}$ and $\alpha_{i} \geq 0$. So, for $x \in \mathbb{R}^{d}, x^{\alpha}=x_{1}^{\alpha_{1}} \cdots x_{d}^{\alpha_{d}}$ and $\partial_{x}^{\alpha}=\partial_{x_{1}}^{\alpha_{1}} \cdots \partial_{x_{d}}^{\alpha_{d}}$, where $\partial_{x_{j}}^{\alpha_{j}}=\left(\frac{\partial}{\partial x_{j}}\right)^{\alpha_{j}}$.

$$
E(x, t)=(4 \pi t)^{-\frac{d}{2}} e^{-\frac{x^{2}}{4 t}}, x \in \mathbb{R}^{d}, 0<t<+\infty,
$$

is called heat kernel and has the following properties:

- $E(x, t) \in \mathcal{S}\left(\mathbb{R}_{x}^{d}\right), t>0$,

- $\int_{\mathbb{R}^{d}} E(x, t) d x=1, t>0$,

- $\left(\frac{\partial}{\partial t}-\Delta\right) E(x, t)=0, x \in \mathbb{R}^{d}, 0<t<\infty$.

Moreover we obtain the following estimate on the heat kernel $E(x, t)$ :

Proposition 1. For any $\alpha \in \mathbb{Z}_{+}^{d}$, we have

$$
\left|\partial_{x}^{\alpha} E(x, t)\right| \leq E(x, t)(\alpha !)^{\frac{1}{2}}(2 t)^{-|\alpha|}(1+|x|)^{\alpha}, x \in \mathbb{R}^{d}, 0<t \leq \frac{1}{2} .
$$

Proof. Since $\hat{E}(\xi, t)=e^{-t \xi^{2}}$, we have

$$
E(x, t)=\left(\frac{1}{2 \pi}\right)^{d} \int_{\mathbb{R}^{d}} e^{-t \xi^{2}} e^{i x \cdot \xi} d \xi
$$

Therefore we obtain

$$
\begin{aligned}
\partial_{x}^{\alpha} E(x, t) & =\partial_{x}^{\alpha}\left(\frac{1}{2 \pi}\right)^{d} \int_{\mathbb{R}^{d}} e^{-t \xi^{2}} e^{i x \cdot \xi} d \xi \\
& =\left(\frac{1}{2 \pi}\right)^{d} \int_{\mathbb{R}^{d}} e^{-t \xi^{2}}(i \xi)^{\alpha} e^{i x \cdot \xi} d \xi
\end{aligned}
$$

Put $\zeta=\xi+i \eta, \xi, \eta \in \mathbb{R}^{d}$. Then by Cauchy's integral theorem,

$$
\begin{aligned}
\partial_{x}^{\alpha} E(x, t) & =\left(\frac{1}{2 \pi}\right)^{d} \int_{\mathbb{R}^{d}} e^{-t \xi^{2}}(i \xi)^{\alpha} e^{i x \cdot \xi} d \xi \\
& =\left(\frac{1}{2 \pi}\right)^{d} \int_{\mathbb{R}^{d}} e^{-t(\xi+i \eta)^{2}}\{i(\xi+i \eta)\}^{\alpha} e^{i x \cdot(\xi+i \eta)} d \xi
\end{aligned}
$$


So we have

$$
\begin{aligned}
\left|\partial_{x}^{\alpha} E(x, t)\right| & =\left|\left(\frac{1}{2 \pi}\right)^{d} \int_{\mathbb{R}^{d}} e^{-t(\xi+i \eta)^{2}}\{i(\xi+i \eta)\}^{\alpha} e^{i x \cdot(\xi+i \eta)} d \xi\right| \\
& \leq\left(\frac{1}{2 \pi}\right)^{d} \int_{\mathbb{R}^{d}} e^{-t\left(\xi^{2}-\eta^{2}\right)}(|\xi|+|\eta|)^{\alpha} e^{-x \cdot \eta} d \xi \\
& =e^{t \eta^{2}-x \cdot \eta}\left(\frac{1}{2 \pi}\right)^{d} \int_{\mathbb{R}^{d}} e^{-t \xi^{2}}(|\xi|+|\eta|)^{\alpha} d \xi \\
& =e^{t\left(\eta-\frac{x}{2 t}\right)^{2}-\frac{x^{2}}{4 t}}\left(\frac{1}{2 \pi}\right)^{d} \int_{\mathbb{R}^{d}} e^{-t \xi^{2}}(|\xi|+|\eta|)^{\alpha} d \xi
\end{aligned}
$$

Now we put $\eta=\frac{x}{2 t}$. Then we obtain

$$
\left|\partial_{x}^{\alpha} E(x, t)\right| \leq e^{-\frac{x^{2}}{4 t}}\left(\frac{1}{2 \pi}\right)^{d} \int_{\mathbb{R}^{d}} e^{-t \xi^{2}}\left(|\xi|+\left|\frac{x}{2 t}\right|\right)^{\alpha} d \xi
$$

On the other hand, we find

$$
\begin{aligned}
\int_{\mathbb{R}^{d}} e^{-t \xi^{2}}\left(|\xi|+\left|\frac{x}{2 t}\right|\right)^{\alpha} d \xi & =\sum_{\beta \leq \alpha}\left(\begin{array}{c}
\alpha \\
\beta
\end{array}\right)\left|\frac{x}{2 t}\right|^{\alpha-\beta} \int_{\mathbb{R}^{d}} e^{-t \xi^{2}}|\xi|^{\beta} d \xi \\
& =2^{d} \sum_{\beta \leq \alpha}\left(\begin{array}{c}
\alpha \\
\beta
\end{array}\right)\left|\frac{x}{2 t}\right|^{\alpha-\beta} \int_{(0, \infty)^{d}} e^{-t \xi^{2} \xi^{\beta} d \xi}
\end{aligned}
$$

Since

$$
\int_{(0, \infty)^{d}} e^{-t \xi^{2}} \xi^{\beta} d \xi=\left(\frac{1}{2}\right)^{d}\left(\frac{1}{\sqrt{t}}\right)^{|\beta|+d} \prod_{j=1}^{d} \Gamma\left(\frac{\beta_{j}+1}{2}\right)
$$

where $\Gamma(s)$ is Euler gamma function. By (2.1) and (2.2), we have

$$
\left|\partial_{x}^{\alpha} E(x, t)\right| \leq e^{-\frac{x^{2}}{4 t}}\left(\frac{1}{2 \pi}\right)^{d} \sum_{\beta \leq \alpha}\left(\begin{array}{l}
\alpha \\
\beta
\end{array}\right)\left|\frac{x}{2 t}\right|^{\alpha-\beta}\left(\frac{1}{\sqrt{t}}\right)^{|\beta|+d} \prod_{j=1}^{d} \Gamma\left(\frac{\beta_{j}+1}{2}\right) .
$$

By a direct calculation, we have

$$
\prod_{j=1}^{d} \Gamma\left(\frac{\beta_{j}+1}{2}\right) \leq(\beta !)^{\frac{1}{2}}\left(\frac{1}{2}\right)^{\frac{|\beta|}{2}} \pi^{\frac{d}{2}}
$$


So we obtain

$$
\begin{aligned}
\left|\partial_{x}^{\alpha} E(x, t)\right| & \leq e^{-\frac{x^{2}}{4 t}}\left(\frac{1}{2 \pi}\right)^{d} \sum_{\beta \leq \alpha}\left(\begin{array}{c}
\alpha \\
\beta
\end{array}\right)\left|\frac{x}{2 t}\right|^{\alpha-\beta}\left(\frac{1}{\sqrt{t}}\right)^{|\beta|+d}(\beta !)^{\frac{1}{2}}\left(\frac{1}{2}\right)^{\frac{|\beta|}{2}} \pi^{\frac{d}{2}} \\
& \leq e^{-\frac{x^{2}}{4 t}}\left(\frac{1}{2 \pi}\right)^{d} \sum_{\beta \leq \alpha}\left(\begin{array}{c}
\alpha \\
\beta
\end{array}\right)\left|\frac{x}{2 t}\right|^{\alpha-\beta}\left(\frac{1}{\sqrt{t}}\right)^{|\beta|+d}(\alpha !)^{\frac{1}{2}}\left(\frac{1}{2}\right)^{\frac{|\beta|}{2}} \pi^{\frac{d}{2}} \\
& =\left(\frac{1}{\sqrt{4 \pi t}}\right)^{d} e^{-\frac{x^{2}}{4 t}}(\alpha !)^{\frac{1}{2}} \sum_{\beta \leq \alpha}\left(\begin{array}{c}
\alpha \\
\beta
\end{array}\right)\left|\frac{x}{2 t}\right|^{\alpha-\beta}\left(\frac{1}{\sqrt{2 t}}\right)^{|\beta|} \\
& =E(x, t)(\alpha !)^{\frac{1}{2}}\left(\left|\frac{x}{2 t}\right|+\frac{1}{\sqrt{2 t}}\right)^{\alpha} \\
& =E(x, t)(\alpha !)^{\frac{1}{2}}\left(\frac{1}{2 t}\right)^{|\alpha|}(|x|+\sqrt{2 t})^{\alpha} .
\end{aligned}
$$

Therefore we have

$$
\left|\partial_{x}^{\alpha} E(x, t)\right| \leq E(x, t)(\alpha !)^{\frac{1}{2}}\left(\frac{1}{2 t}\right)^{|\alpha|}(1+|x|)^{\alpha}, 0<t \leq \frac{1}{2}
$$

which proves the Proposition 1.

Remark 1. The estimate in Proposition 1 is a little bit different from Matsuzawa's result in [5].

Definition 1. Let $K$ be a compact set of $\mathbb{R}^{d}$ and $\mathcal{A}(K)$ be the space of all real analytic functions in some neighborhood of $K$. That is,

$\mathcal{A}(K)=\left\{\varphi \in C^{\infty}\right.$ in a neighborhood of $K \mid \exists C>0, \exists h>0$ such that

$$
\left.\sup _{x \in K, \alpha} \frac{\left|\partial^{\alpha} \varphi(x)\right|}{h^{|\alpha|} \alpha !} \leq C\right\} .
$$

For $h>0$, we put

$$
\begin{gathered}
\mathcal{A}_{h}(K)=\left\{\varphi \in C^{\infty} \text { in a neighborhood of } K \mid \exists C>0\right. \text { such that } \\
\left.\|\varphi\|_{\mathcal{A}_{h}(K)}=\sup _{x \in K, \alpha} \frac{\left|\partial^{\alpha} \varphi(x)\right|}{h^{|\alpha|} \alpha !} \leq C\right\} .
\end{gathered}
$$

Then $\mathcal{A}(K)=\lim _{\substack{-\rightarrow \rightarrow \infty \\ h \rightarrow \infty}} \mathcal{A}_{h}(K) . \mathcal{A}_{h}(K)$ is Banach space and $\mathcal{A}(K)$ is DFS-space.

Remark 2. This norm $\|\cdot\|_{\mathcal{A}_{h}(K)}$ is equivalent to the following norm $\|\cdot\|_{\omega}$. Let $\omega$ be a complex neighborhood of $K$. For any $\varphi \in \mathcal{O}(\omega) \cap C(\bar{\omega})$,

$$
\|\varphi\|_{\omega}=\sup _{\omega}|\varphi(\zeta)| \text {. }
$$

By Proposition 1, we immediately obtain the following Proposition 2:

Proposition 2. $E(x, t) \in \mathcal{A}_{h}(K)$. 
Definition 2. $\mathcal{A}^{\prime}(K)$ denotes the dual space of $\mathcal{A}(K)$ and we call the element of $\mathcal{A}^{\prime}(K)$ hyperfunctions supported by $K$.

We obtain the following result.

Theorem 2. Let $U(x, t) \in C^{\infty}\left(\mathbb{R}^{d} \times(0, \infty)\right)$ satisfy the following conditions:

(i) $\left(\frac{\partial}{\partial t}-\Delta\right) U(x, t)=0$,

(ii) $\forall \epsilon>0, \exists C_{\epsilon}>0$ s.t. $|U(x, t)| \leq C_{\epsilon} e^{\varepsilon / t}, 0<t<1, x \in \mathbb{R}^{d}$,

(iii) $\forall \delta>0, U(x, t) \rightarrow 0$ uniformly in $\left\{x \in \mathbb{R}^{d} \mid \operatorname{dist}(x, K) \geq \delta\right\}$ as $t \rightarrow 0$.

Then $U(x, t)$ has the following asymptotic expansion:

$$
U(x, t) \sim \sum_{k=0}^{\infty} \frac{t^{k}}{k !} \Delta_{x}^{k} u, \quad\left(u \in \mathcal{A}^{\prime}(K), \text { where } u=\lim _{t \rightarrow 0} U(x, t)\right) .
$$

That is, for any entire function $\varphi$,

$$
\lim _{t \rightarrow 0+}\left|\int_{\mathbb{R}^{d}} U(x, t) \chi(x) \varphi(x)-\left\langle\sum_{k=0}^{\frac{N}{2}} \frac{t^{k}}{k !} \Delta_{x}^{k} u, \chi \varphi\right\rangle\right| t^{-\frac{N}{2}}=0,
$$

where $\chi(x) \in C_{0}^{\infty}\left(\mathbb{R}^{d}\right)$ and $\chi=1$ in a neighborhood of $K$.

To prove Theorem 2, we need the two lemmas as follows:

Lemma 1. Let $\chi \in C_{0}^{\infty}$ such as $\chi=1$ in a neighborhood of $K$ and $\varphi$ be entire functions. Then $\partial^{\alpha}(\chi \varphi) \in \mathcal{A}(K)$ for any $\alpha \in \mathbb{Z}_{+}^{d}$.

Proof.

$$
\partial^{\alpha}(\chi \varphi)=\sum_{|\beta| \leq \alpha}\left(\begin{array}{l}
\alpha \\
\beta
\end{array}\right) \partial^{\beta} \chi \partial^{\alpha-\beta} \varphi
$$

Since $\partial^{\beta} \chi \partial^{\alpha-\beta} \varphi \in \mathcal{A}(K)$, we obtain $\partial^{\alpha}(\chi \varphi) \in \mathcal{A}(K)$.

Lemma 2. Let $g=\chi \varphi$ be in $\mathcal{A}_{h}(K)$ and $t>0$, where $\chi \in C_{0}^{\infty}\left(\mathbb{R}^{d}\right)$ such as $\chi=1$ in a neighborhood of $K$ and $\varphi$ be entire functions. Then

$$
\int_{\mathbb{R}^{d}} e^{-z^{2}} z^{\alpha}\left\{\int_{0}^{1}(1-\theta)^{N} \partial_{y}^{\alpha} g(y+\sqrt{4 t} z \theta) d \theta\right\} d z
$$

is in $\mathcal{A}_{h}(K)$.

Proof. For $y \in K$, let given $h>0$ be fixed. If $g \in \mathcal{A}_{h}(K)$, then by Lemma 1 , $\partial^{\alpha} g \in \mathcal{A}_{h}(K)$ for any $\alpha \in \mathbb{Z}_{+}^{d}$. If we put $G(y)=\partial^{\alpha} g(y+\sqrt{4 t} z \theta)$, then $G(y) \in \mathcal{A}_{h}(K)$. So there exists constant $C_{G}$ such that

$$
\sup _{y \in K, \beta} \frac{\left|\partial_{y}^{\beta} G(y)\right|}{h^{|\beta|} \beta !} \leq C_{G} .
$$


Therefore

$$
\begin{array}{r}
\frac{\left|\partial^{\beta} \int_{\mathbb{R}^{d}} e^{-z^{2}} z^{\alpha}\left\{\int_{0}^{1}(1-\theta)^{N} G(y) d \theta\right\} d z\right|}{h^{|\beta|} \beta !} \\
\leq C_{G} \int_{\mathbb{R}^{d}} e^{-z^{2}} z^{\alpha} d z=C_{G} \prod_{j=1}^{d} \Gamma\left(\frac{1}{2}\left(\alpha_{j}+1\right)\right) .
\end{array}
$$

So we obtain

$$
\int_{\mathbb{R}^{d}} e^{-z^{2}} z^{\alpha}\left\{\int_{0}^{1}(1-\theta)^{N} \partial_{y}^{\alpha} g(y+\sqrt{4 t} z \theta) d \theta\right\} d z \in \mathcal{A}_{h}(K) .
$$

This proves the lemma.

Proof of Theorem 2. By Matsuzawa's result [4], there uniquely exists $u \in \mathcal{A}^{\prime}(K)$ such that $U(x, t)=(u * E)(x, t)$. Hence, for any entire functions $\varphi$,

$$
\begin{aligned}
\langle U(x, t), \chi(x) \varphi(x)\rangle & =\langle(u * E)(x, t), \chi(x) \varphi(x)\rangle \\
& =\left\langle u_{y}, E(x, t) * \chi \varphi\right\rangle \\
& =\left\langle u_{y}, \int_{\mathbb{R}^{d}} \pi^{-\frac{d}{2}} e^{-z^{2}} \chi(y+\sqrt{4 t} z) \varphi(y+\sqrt{4 t} z) d z\right\rangle .
\end{aligned}
$$

Put $g(y+\sqrt{4 t} z)=\chi(y+\sqrt{4 t} z) \varphi(y+\sqrt{4 t} z)$. Then by Taylor's formula,

$$
\begin{aligned}
& \int_{\mathbb{R}^{d}} \pi^{-\frac{d}{2}} e^{-z^{2}} g(y+\sqrt{4 t} z) d z \\
= & \pi^{-\frac{d}{2}} \sum_{|\alpha|=0}^{N} \frac{\partial^{\alpha} g(y)}{\alpha !}(\sqrt{4 t})^{|\alpha|} \int_{\mathbb{R}^{d}} e^{-z^{2}} z^{\alpha} d z \\
& +\int_{\mathbb{R}^{d}} \pi^{\frac{-d}{2}} e^{-z^{2}}\left\{\sum_{|\alpha|=N+1} \frac{N+1}{\alpha !}(\sqrt{4 t} z)^{\alpha} \int_{0}^{1}(1-\theta)^{N} \partial^{\alpha} g(y+\sqrt{4 t} z \theta) d \theta\right\} d z .
\end{aligned}
$$

So we obtain

$$
\begin{aligned}
& \left|\langle U(x, t), \chi \varphi\rangle-\left\langle\sum_{k=0}^{\frac{N}{2}} \frac{t^{k}}{k !} \Delta^{k} u, \chi \varphi\right\rangle\right| t^{-\frac{N}{2}} \\
= & \left|\left\langle u_{y}, \int_{\mathbb{R}^{d}} \pi^{\frac{-d}{2}} e^{-z^{2}}\left\{\sum_{|\alpha|=N+1} \frac{N+1}{\alpha !}(\sqrt{4 t} z)^{\alpha} \int_{0}^{1}(1-\theta)^{N} \partial^{\alpha} g(y+\sqrt{4 t} z \theta) d \theta\right\} d z\right\rangle\right| t^{-\frac{N}{2}} .
\end{aligned}
$$

Therefore, by Lemma 5 and the continuity of $u$, we have

$$
\left|\langle U(x, t), \chi \varphi\rangle-\left\langle\sum_{k=0}^{\frac{N}{2}} \frac{t^{k}}{k !} \Delta^{k} u, \chi \varphi\right\rangle\right| t^{-\frac{N}{2}} \leq C t^{\frac{N+1}{2}} t^{-\frac{N}{2}}=C t^{\frac{1}{2}} \rightarrow 0, \text { as } t \rightarrow 0 .
$$




\section{Asymptotic expansions of the solutions to the heat equations with Fourier-hyperfunctions initial data}

We will derive asymptotic expansions of the solutions to the heat equations with Fourier hyperfunctions initial data in this section.

At first, we define the space of test functions $\mathcal{F}$ of Fourier-hyperfunctions as follows:

Definition 3. For $h, k \in(0, \infty)^{d}$,

$$
\begin{aligned}
\mathcal{F}_{h, k}=\left\{\varphi \in C^{\infty}\left(\mathbb{R}^{d}\right) \mid\right. & \forall \delta, \rho \in(1, \infty)^{d}, \exists C_{\delta, \rho} \geq 0 \text { such that } \\
& \left.\left|\partial^{\beta} \varphi(x)\right| \leq C_{\delta, \rho}(\rho h)^{\beta} \beta ! \exp (-k \delta|x|), \forall \beta \in \mathbb{Z}_{+}^{d}\right\} .
\end{aligned}
$$

- $\mathcal{F}_{h, k}$ is a Fréchet space by semi norm

$$
\|\varphi\|_{\delta, \rho}=\sup _{x, \beta} \frac{\left|\partial^{\beta} \varphi(x)\right| \exp (\delta k)|x|}{(\rho h)^{\beta} \beta !}
$$

and $\mathcal{F}$ is LF-space. $\left(\mathcal{F}=\lim _{\substack{\longrightarrow \rightarrow \infty \\ k \rightarrow 0}} \mathcal{F}_{h, k}\right)$.

Remark 3. It is known that $\mathcal{F} \cong \mathcal{S}_{1}^{1}[6]$, where $\mathcal{S}_{1}^{1}$ is the Gel'fand-Shilov space $[2]$.

On the other hand, J. Chung, S.-Y. Chung, and D. Kim characterized $\mathcal{F}$ via Fourier transform as follows [1]:

Proposition 3 ([1]).

$$
\begin{aligned}
\mathcal{F}=\left\{\varphi \in C^{\infty}\left(\mathbb{R}^{d}\right) \mid\right. & \sup |\varphi(x)| \exp (m|x|)<\infty \\
& \sup |\hat{\varphi}(\xi)| \exp (l|\xi|)<\infty \text { for some } l, m>0\} .
\end{aligned}
$$

Definition 4. $\mathcal{F}^{\prime}$ denotes the dual space of $\mathcal{F}$ and the elements of $\mathcal{F}^{\prime}$ are called Fourier hyperfunctions.

Proposition 4. Let $u$ be a linear form from $\mathcal{F}$ to $\mathbb{C}$. Then the following statements (i) and (ii) are equivalent.

(i) $u \in \mathcal{F}^{\prime}\left(\mathbb{R}^{d}\right)$.

(ii) $\forall h, k \in(0, \infty)^{d}, \exists C_{h, k}>0$ s.t. $|\langle u, \varphi\rangle| \leq C_{h, k}\|\varphi\|_{\delta, \rho}, \forall \varphi \in \mathcal{F}_{h, k}\left(\mathbb{R}^{d}\right)$.

We obtain the following asymptotic expansions of the solutions to the heat equations with Fourier-hyperfunctions initial data:

Theorem 3. Let $U(x, t) \in C^{\infty}\left(\mathbb{R}^{d} \times(0, \infty)\right)$ satisfy the following conditions:

(i) $\left(\frac{\partial}{\partial t}-\Delta\right) U(x, t)=0$,

(ii) $\forall \epsilon>0, \exists C_{\epsilon}>0$ s.t. $|U(x, t)| \leq C_{\epsilon} \exp [\epsilon(1 / t+|x|)], 0<t<1$, $x \in \mathbb{R}^{d}$. 
ASYMPTOTIC EXPANSIONS OF THE SOLUTIONS TO THE HEAT EQUATIONS 563

Then $U(x, t)$ has the following asymptotic expansion:

$$
U(x, t) \sim \sum_{m=0}^{\infty} \frac{t^{m}}{m !} \Delta_{x}^{m} u,\left(u \in \mathcal{F}^{\prime}\left(\mathbb{R}^{d}\right), \text { where } u=\lim _{t \rightarrow 0} U(x, t)\right) .
$$

That is,

$$
\lim _{t \rightarrow 0+}\left|\int_{\mathbb{R}^{d}} U(x, t) \varphi(x) d x-\left\langle\sum_{m=0}^{\frac{N}{2}} \frac{t^{m}}{m !} \Delta_{x}^{m} u, \varphi\right\rangle\right| t^{-\frac{N}{2}}=0, \varphi \in \mathcal{F}\left(\mathbb{R}^{d}\right) .
$$

To show this, we need the following lemma:

Lemma 3. Let $\varphi$ be in $\mathcal{F}_{h, k}\left(\mathbb{R}^{d}\right)$ and $t>0$. Then

$$
\int_{\mathbb{R}^{d}} e^{-z^{2}} z^{\alpha}\left\{\int_{0}^{1}(1-\theta)^{N} \partial_{y}^{\alpha} \varphi(y+\sqrt{4 t} z \theta) d \theta\right\} d z
$$

belongs to $\mathcal{F}_{h, k}\left(\mathbb{R}^{d}\right)$.

Proof.

$$
\frac{\left|\partial_{y}^{\beta} \int_{\mathbb{R}^{d}} e^{-z^{2}} z^{\alpha}\left\{\int_{0}^{1}(1-\theta)^{N} \partial_{y}^{\alpha} \varphi(y+\sqrt{4 t} z \theta) d \theta\right\} d z\right| \exp (\delta k)|y|}{(\rho h)^{\beta} \beta !} .
$$

Since $\varphi \in \mathcal{F}_{h, k}, \partial_{y}^{\alpha} \varphi \in \mathcal{F}_{h, k}[2]$. Put $g(y+\sqrt{4 t} z \theta)=\partial_{y}^{\alpha} \varphi(y+\sqrt{4 t} z \theta)$.

$$
\begin{aligned}
(3.1) & =\frac{\left|\partial_{y}^{\beta} \int_{\mathbb{R}^{d}} e^{-z^{2}} z^{\alpha}\left\{\int_{0}^{1}(1-\theta)^{N} g(y+\sqrt{4 t} z \theta) d \theta\right\} d z\right| \exp (\delta k)|y|}{(\rho h)^{\beta} \beta !} \\
& \leq \frac{\int_{\mathbb{R}^{d}} e^{-z^{2}}|z|^{\alpha}\left\{\int_{0}^{1}|1-\theta|^{N}\left|\partial_{y}^{\beta} g(y+\sqrt{4 t} z \theta)\right| d \theta\right\} d z \exp (\delta k)|y|}{(\rho h)^{\beta} \beta !} \\
(3.2) \quad & =\int_{\mathbb{R}^{d}} e^{-z^{2}|z|^{\alpha}\left\{\int_{0}^{1}|1-\theta|^{N} \frac{\left|\partial_{y}^{\beta} g(y+\sqrt{4 t} z \theta)\right|}{(\rho h)^{\beta} \beta !} d \theta\right\} d z \exp (\delta k)|y| .}
\end{aligned}
$$

By $g \in \mathcal{F}_{h, k}$,

$$
\frac{\left|\partial_{y}^{\beta} g(y+\sqrt{4 t} z \theta)\right|}{(\rho h)^{\beta} \beta !} \leq C_{\delta, \rho} \exp (-(\delta k)|y+\sqrt{4 t} z \theta|) .
$$


So we have

$$
\begin{aligned}
(3.2) & \leq \int_{\mathbb{R}^{d}} e^{-z^{2}}|z|^{\alpha} C_{\delta, \rho} \exp (-(\delta k)|y+\sqrt{4 t} z \theta|) \exp (\delta k)|y| d z \\
& \leq C_{\delta, \rho} \int_{\mathbb{R}^{d}} e^{-z^{2}}|z|^{\alpha} \exp ((\delta k) \sqrt{4 t}|z|) d z \\
& \leq C_{\delta, \rho} \int_{\mathbb{R}^{d}} e^{-z^{2}}|z|^{\alpha} \exp (2(\delta k)|z|) d z \\
& \leq C_{\delta, \rho} e^{\delta^{2} k^{2}} \sum_{|l| \leq \alpha}\left(\begin{array}{c}
\alpha \\
l
\end{array}\right)(\delta k)^{\alpha-l+1}\left\{\left(\frac{l}{2}\right)^{l / 2}+\Gamma\left(\frac{1}{2}(l+1)\right)\right\} .
\end{aligned}
$$

Hence we obtain

$$
\int_{\mathbb{R}^{d}} e^{-z^{2}} z^{\alpha}\left\{\int_{0}^{1}(1-\theta)^{N} \partial_{y}^{\alpha} \varphi(y+\sqrt{4 t} z \theta) d \theta\right\} d z \in \mathcal{F}_{h, k} .
$$

This completes the proof of Lemma 3 .

Proof of Theorem 3. By the result in [3], there exists Fourier-hyperfunction $u \in \mathcal{F}$ such that $U(x, t)=(u * E)(x, t)$. For any $\varphi \in \mathcal{F}_{h, k}$,

$$
\langle U(x, t), \varphi\rangle=\langle(u * E)(x, t), \varphi\rangle .
$$

By a similar calculation to the proof of Theorem 2, we have the following equality:

$$
\begin{aligned}
& \left|\langle U(x, t), \varphi\rangle-\sum_{m=0}^{\frac{N}{2}} \frac{t^{m}}{m !}\left\langle\Delta^{m} u, \varphi\right\rangle\right| t^{-\frac{N}{2}} \\
= & \left|\left\langle u_{y}, \pi^{-\frac{d}{2}}(\sqrt{4 t})^{N+1} \sum_{|\alpha|=N+1} \frac{(N+1)}{\alpha !} \int_{\mathbb{R}^{d}} e^{-z^{2}} z^{\alpha}\left\{\int_{0}^{1}(1-\theta)^{N} \partial_{y}^{\alpha} \varphi(y+\sqrt{4 t} z \theta) d \theta\right\} d z\right\rangle\right| t^{-\frac{N}{2}} .
\end{aligned}
$$

We obtain the following estimate by Proposition 4 and Lemma 3. For any $h, k \in(0, \infty)^{d}$,

$$
(3.3) \leq C_{h, k, \delta, \rho, \alpha} t^{\frac{N+1}{2}} t^{-\frac{N}{2}}=C_{h, k, \delta, \rho, \alpha} t^{\frac{1}{2}} \rightarrow 0, t \rightarrow 0 .
$$

This completes the proof of Theorem 3 .

Remark 4. In this connection, we have antecedent results on the asymptotic expansions of the solutions of the heat equations with the tempered distributions and the distributions of exponential growth initial date (see [7]).

\section{References}

[1] J. Chung, S.-Y. Chung, and D. Kim, A characterization for Fourier hyperfunctions, Publ. RIMS, Kyoto Univ. 30 (1994), 203-208.

[2] I. M. Gel'fand and G. E. Shilov, Generalized Functions, Volume 2, Space of Fundamental and Generalized Functions, Academy of Sciences Moscow, U. S. S. R, 1958. 
ASYMPTOTIC EXPANSIONS OF THE SOLUTIONS TO THE HEAT EQUATIONS 565

[3] K. W. Kim, S.-Y. Chung, and D. Kim, Fourier hyperfunctions as the boundary values of smooth solutions of the heat equation, Publ, RIMS, Kyoto Univ. 29 (1993), 289-300.

[4] T. Matsuzawa, A calculus approach to the hyperfunctions I, Nagoya Math. J. 108 (1987), $53-66$.

[5] — A calculus approach to the hyperfunctions II, Trans. Amer. Math. Soc. 313 (1989), no. 2, 619-654.

[6] S. Nagamachi and N. Mugibayashi, Hyperfunction quantum field theory, Commun. Math. Phys. 46 (1976), 119-134.

[7] K. Yoshino and Y. Oka, Asymptotic expansions of the solutions to the heat equations with generalized functions initial value, Complex Analysis and Potential Theory, Proceedings of the Conference Satellite to ICM 2006 (Gebze Institute of Technology, Turkey) (Tahir Aliyev Azeroğlu and Promarz M. Tamrazov eds.), World Scientific Publishing Co. Pte. Ltd (2007), 198-206.

KUnio Yoshino

FACUlty of KnOWLedge EngineERIng

Musashi Institute of Technology

1-28-1 Tamazutsumi, Setagaya-Ku, TOKyo 158-8557, Japan

E-mail address: yoshino@ma.ns.musashi-tech.ac.jp

YASUYUKI OKA

Department of Mathematics

SOPHIA UNIVERSITY

7-1 Kioicho, Chiyoda-Ku, Tokyo 102-8554, Japan

E-mail address: yasuyu-o@hoffman.cc.sophia.ac.jp 